\title{
Stratégies d'apprentissage et exploitation de textes techniques
}

Janet Atlan

\section{OpenEdition}

\section{Journals}

Édition électronique

URL : http://journals.openedition.org/asp/4277

DOI : $10.4000 /$ asp. 4277

ISSN : 2108-6354

\section{Éditeur}

Groupe d'étude et de recherche en anglais de spécialité

\section{Édition imprimée}

Date de publication : 1 octobre 1993

Pagination : 121-137

ISSN : 1246-8185

\section{Référence électronique}

Janet Atlan, «Stratégies d'apprentissage et exploitation de textes techniques », ASp [En ligne],

2 | 1993, mis en ligne le 28 février 2014, consulté le 30 avril 2019. URL : http://

journals.openedition.org/asp/4277 ; DOI : 10.4000/asp.4277

Ce document a été généré automatiquement le 30 avril 2019.

Tous droits réservés 


\title{
Stratégies d'apprentissage et exploitation de textes techniques
}

\author{
Janet Atlan
}

1 Cette communication présente une expérience faite à l'IUT de Nancy II avec des étudiants du Département Informatique. L'expérience avait pour but d'observer et analyser les techniques et donc les stratégies particulières utilisées par les apprenants pour acquérir, manipuler et reproduire de nouvelles données en présence de textes informatiques très techniques en anglais. Pour ce faire, nous avons donné aux apprenants un exercice portant sur ce genre de texte et composé de trois tâches distinctes : la compréhension du texte, la préparation d'un exposé oral à partir de ce texte et la présentation de l'exposé oral devant un auditoire.

\section{Déroulement de l'exercice}

2 Après avoir choisi un texte de présentation de produit informatique dans un journal spécialisé, chaque étudiant devait le lire et le comprendre. Ce texte avait au plus deux pages et ne pouvait pas être un document publicitaire. Ensuite l'étudiant préparait une présentation orale du produit choisi. Enfin, il exécutait cette présentation devant la classe. La présentation durait entre deux et trois minutes et se faisait sans aucune note. Elle était notée par l'enseignant.

3 Le choix de ce genre de textes était dicté par la quantité de vocabulaire spécialisé qu'ils contiennent : acronymes, nombres, unités de mesure, noms propres (voir annexe 1 pour un exemple). Les étudiants disposaient de plusieurs séances en classe étalées sur trois semaines pour faire ce travail. Le fait de présenter sans notes exige de l'étudiant un effort pour utiliser d'autres techniques que la simple lecture d'un texte écrit à l'avance.

4 Cet exercice a été fait avec trois groupes d'étudiants de niveau intermédiaire en première et deuxième année. À la fin de l'exercice, nous avons demandé aux étudiants de remplir un questionnaire portant sur leur façon de travailler pendant les trois phases de l'exercice (voir annexe 2). Les questions étaient posées de façon à expliciter l'approche 
globale des tâches par l'étudiant et l'approche spécifique de certains éléments des tâches (la manipulation et reproduction d'acronymes par exemple).

Les réponses au questionnaire étaient libres avec seulement quelques suggestions données dans les instructions. Les questionnaires ont été remplis en classe à la fin de l'exercice sans que l'enseignant ne donne de conseils et avant que les étudiants ne prennent connaissance de leurs notes. Un total de 43 questionnaires a pu être exploité.

\section{Exploitation du questionnaire}

6 Le questionnaire a été d'abord dépouillé pour dresser la liste de techniques citées. Ensuite chaque technique a été rapprochée d'une stratégie d'apprentissage et un classement par type de stratégie a été construit (voir annexe 3 pour un exemple de dépouillement du questionnaire). Enfin, les résultats ont été examinés afin de repérer l'utilisation éventuelle de stratégies spécifiques au genre de texte exploité. Cet examen portait à la fois sur les stratégies en tant que telles et sur le classement par type de stratégie.

7 Il faut remarquer qu'une même technique peut être le reflet de différentes stratégies selon la tâche à accomplir. Par exemple, la technique de répétition utilisée pour apprendre un mot est une stratégie cognitive. Tandis que la même technique utilisée pour se souvenir d'un texte pour le présenter est une stratégie métacognitive de préparation. Par conséquent, le classement dans une stratégie donnée tient compte à la fois de la technique citée et de la tâche en question.

8 Il faut aussi signaler que les étudiants ont souvent répondu « idem » ou « voir question $n^{\circ}$ $\mathrm{X}$ » plutôt que de détailler leurs techniques quand ils estimaient qu'une question donnée était semblable aux autres dans la série. Dans ce cas nous avons reporté les techniques déjà citées comme réponse à la question. Par contre, si l'étudiant a répondu «je ne sais pas » ou si la réponse était incompréhensible, nous n'avons rien relevé.

\section{Les stratégies d'apprentissage}

9 Les stratégies d'apprentissage sont des mesures prises par l'apprenant pour acquérir, stocker et réutiliser de nouvelles données (Oxford \& Crookall 1989 : 404). Le classement que nous avons retenu pour ce travail est celui de O'Malley et Chamot (1990:119-20, 126, 137-39) et les traductions françaises des termes sont pour la plupart dues à Narcy (1990 115-16).

10 Il y a trois grands types de stratégies d'apprentissage. Les stratégies métacognitives font partie des stratégies «indirectes » et sont l'indication d'une réflexion sur les processus d'apprentissage. Les stratégies socio-affectives sont aussi « indirectes ». Elles sont moins nombreuses et moins utilisées que celles des deux autres catégories mais peuvent être très efficaces. Elles indiquent un processus d'interaction, soit avec autrui, soit avec soimême. Les stratégies cognitives, appelées aussi "directes", sont des actions sur la matière linguistique (voir annexe 4 pour les classifications et définitions des stratégies d'apprentissage).

11 Chamot et Kupper (1989: 19) ont trouvé que tous les apprenants d'une langue, quel que soit leur degré de succès, peuvent contrôler cognitivement leur apprentissage et donc décrire leurs processus mentaux. Cependant, le type de stratégie utilisée peut varier selon 
le niveau de l'apprenant et la tâche à exécuter (Gardner \& MacIntyre 1992 : 217). De façon générale, les apprenants utilisent les stratégies cognitives plus que les métacognitives et les stratégies socio-affectives sont utilisées le moins souvent.

Par ailleurs, O'Malley et Chamot (1990:122) ont trouvé que certaines tâches telles l'apprentissage de vocabulaire et de prononciation ou l'exécution d'exercices à l'oral suscitent l'utilisation de plus de stratégies d'apprentissage que d'autres. Et les tâches pour lesquelles les apprenants citent le plus petit nombre de stratégies sont la compréhension orale, la présentation orale et la communication fonctionnelle.

Finalement, Chamot et Kupper (1989: 14) ont remarqué que les stratégies cognitives les plus utilisées sont celles demandant un traitement mental simple par exemple la répétition, la traduction ou le transfert. Par contre, les stratégies cognitives exigeant un traitement mental complexe ou de processus cognitifs actifs (par exemple, l'association, l'élaboration ou l'image mentale) sont moins fréquentes (voir annexe 4 pour la définition de ces stratégies). De plus, les apprenants de niveau bas et intermédiaire utilisent presque uniquement des stratégies cognitives simples.

\section{L'analyse des résultats}

Une première analyse des résultats globaux (type de stratégie pour chaque tâche, fig. 1) montre que les stratégies métacognitives ont été utilisées de façon égale pour toutes les tâches. Les stratégies cognitives ont été utilisées le plus souvent et les socio-affectives le moins souvent, conformément aux résultats des recherches citées plus haut. La fréquence plus élevée de stratégies socio-affectives lors de la phase de compréhension de texte traduit le fait que les étudiants ont souvent demandé des renseignements à des tiers quand ils ne comprenaient pas.

Figure 1. Résultats globaux du questionnaire

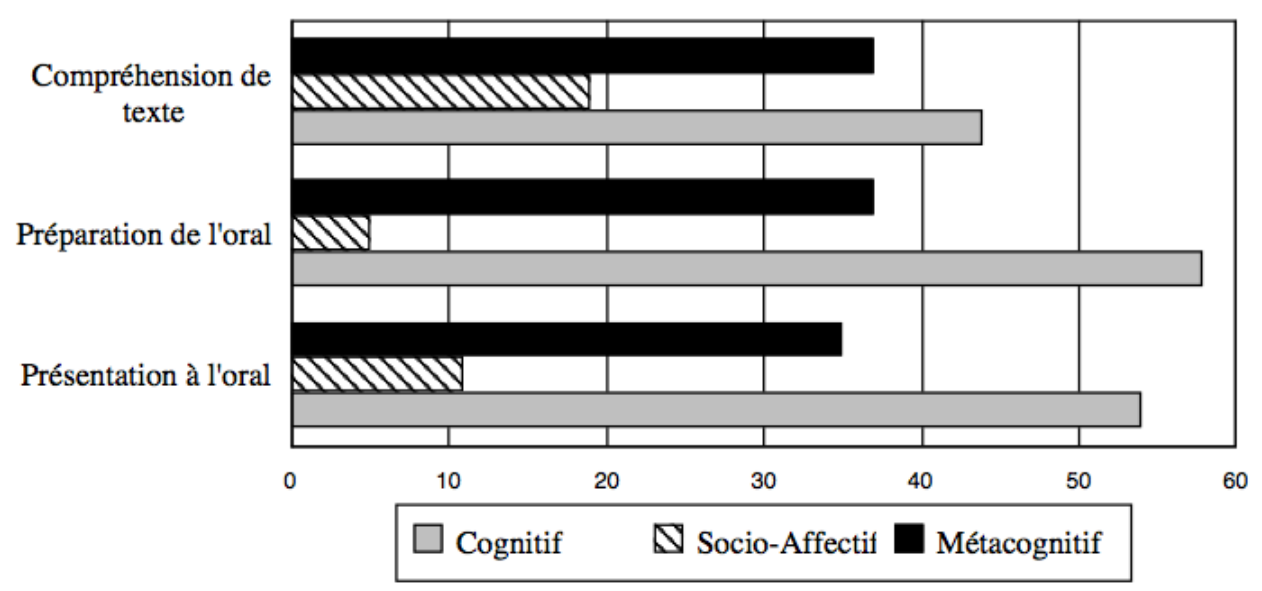

Ensuite nous avons fait une analyse détaillée pour chaque tâche tenant compte du type de questions posées. La première analyse concerne l'approche spécifique de certains éléments de la tâche, à savoir la compréhension, manipulation et reproduction des mots, des acronymes, des unités de mesure, des noms propres et des nombres et dimensions.

Les stratégies utilisées pour cette approche spécifique lors de la lecture et compréhension du texte (voir fig. 2) montrent encore une fois l'effet de la demande de renseignements à 
des tiers. Il semblerait en effet que la tendance était de demander des renseignements sans trier les informations au préalable par l'utilisation de stratégies métacognitives.

La figure 3 montre la proportion de différents types de stratégies utilisés lors de la préparation de la présentation orale. Comme on pourrait s'y attendre, il y a une forte proportion de stratégies cognitives citées pour la rétention des acronymes (les réciter, les écrire, apprendre le sens, apprendre les lettres, etc.). Nous pensons que le rapport stratégies métacognitives/stratégies cognitives pour les nombres et dimensions aurait été semblable à celui des acronymes s'il n'y avait pas eu beaucoup de non-réponses à cette rubrique.

Figure 2. Lecture et compréhension du texte. Tâche : acquérir de nouvelles données

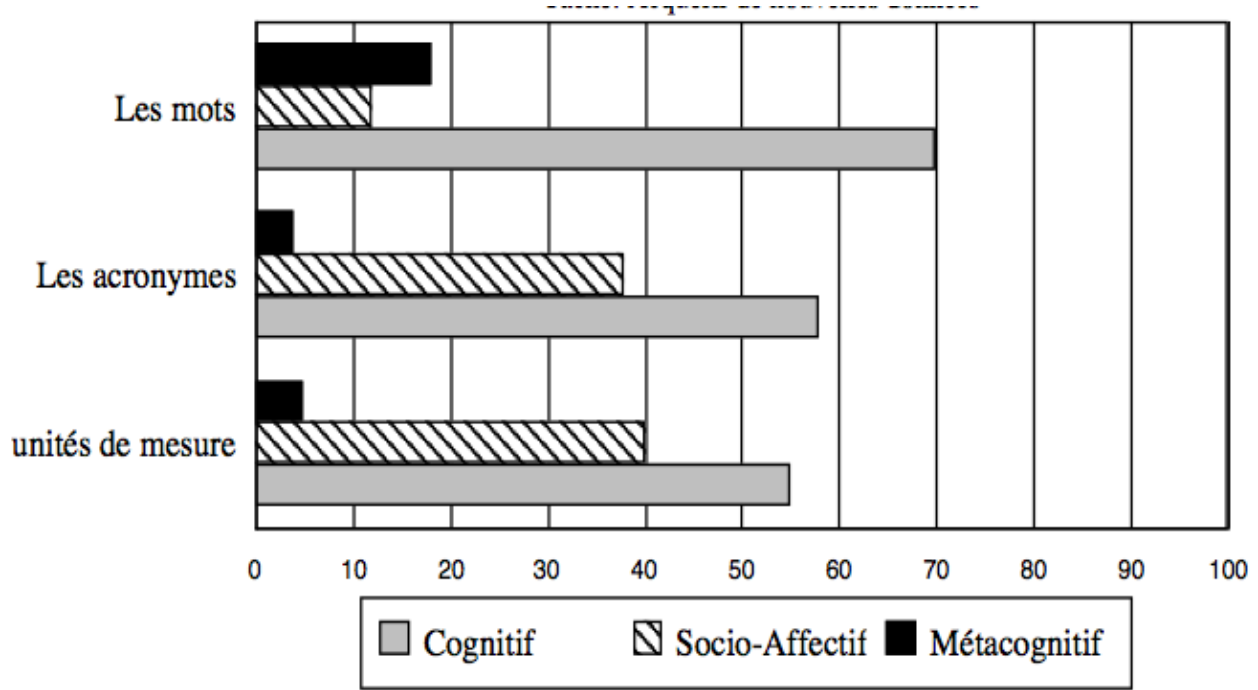

Figure 3. Préparation de l'oral. Tâche : manipuler les nouvelles données

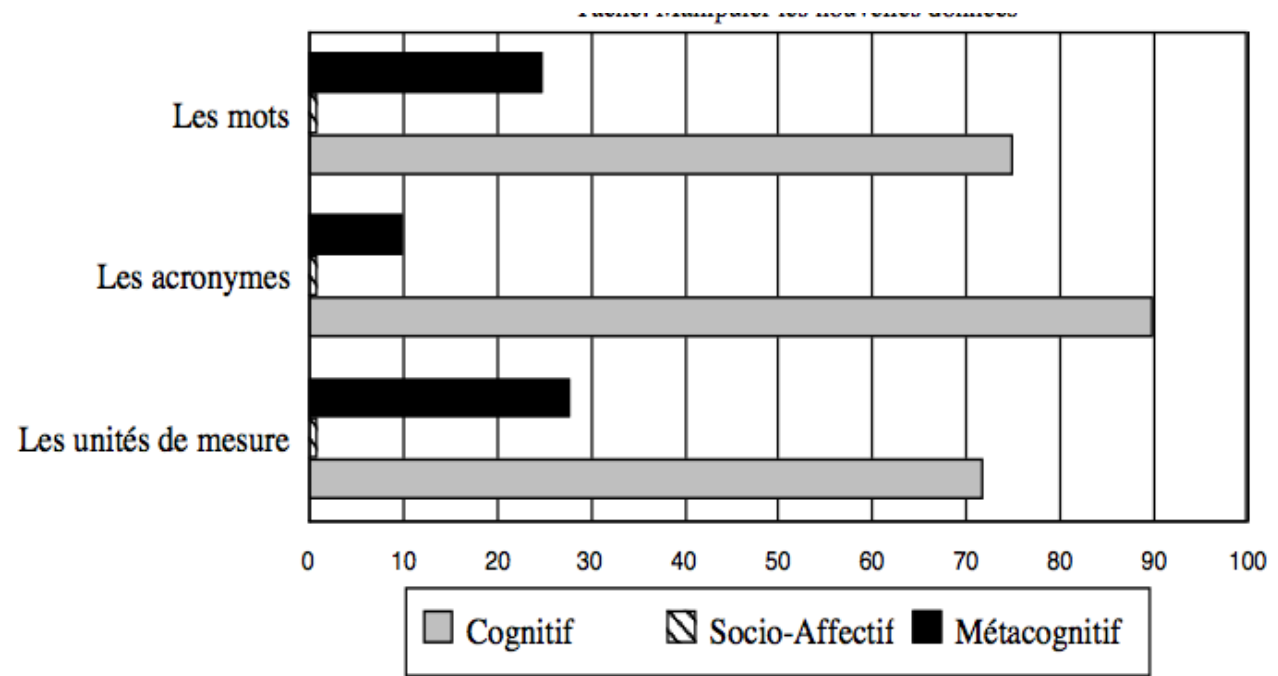

Les apprenants ont pu citer un nombre considérable de techniques utilisées pendant la phase de présentation à l'oral (voir fig.4), contrairement à ce qui est rapporté dans O'Malley et Chamot (1990). Mais il est vrai qu'un certain nombre de stratégies métacognitives citées se rapportaient plutôt à la préparation qu'à la présentation orale 
(par exemple "réciter le texte avant de passer »). On peut noter la forte proportion de stratégies cognitives utilisées ici, surtout pour se souvenir des nombres. Ceci n'est pas seulement dû à l'apprentissage par cœur comme nous le verrons plus loin.

Maintenant nous allons examiner les stratégies utilisées lors de l'approche globale de chacune des trois tâches. Nous avons appelé ces stratégies d'approche globale « d'aide et de conseil » car elles ont été décrites comme une façon de s'aider soi-même ou comme des conseils à des tiers pour faire le même travail. Il nous semble, en effet, que les conseils donnés à autrui sont des stratégies potentielles pour soi-même et peuvent êtres comptabilisées comme telles. La figure 5 montre la proportion de chaque type de stratégie utilisée pour chacune des tâches. La figure contient aussi un rappel des résultats trouvés lors de l'analyse globale des résultats : approche globale et approche spécifique confondues.

Figure 4. Présentation à l'oral. Tâche : reproduire les nouvelles données

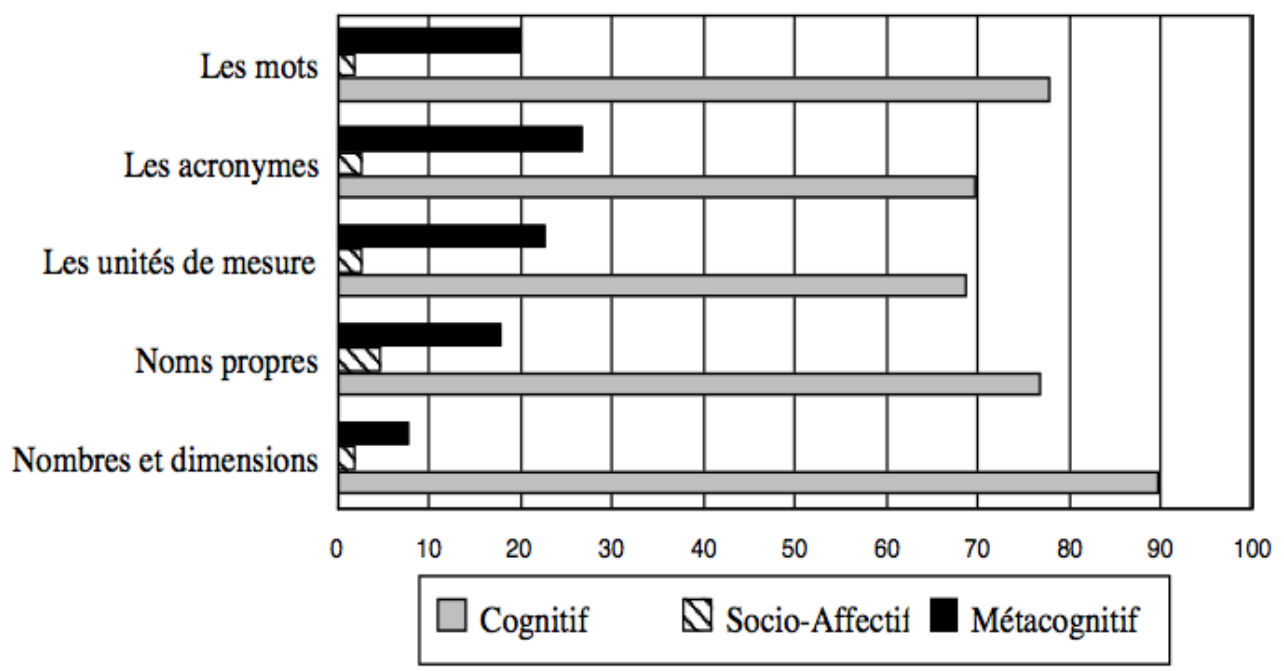

Pendant les phases de compréhension et de préparation, on peut noter une démarche nettement plus métacognitive lorsqu'il s'agit de regarder la tâche dans sa totalité. On peut noter aussi que l'évolution des stratégies pendant ces deux phases est semblable à celle trouvée pour les résultats globaux. Le fait que les stratégies cognitives citées pour l'approche globale soient toujours nettement moins nombreuses nous rappelle que les techniques citées lors de l'approche spécifique étaient surtout du type cognitif.

21 Ce qui est frappant dans la figure 5 est la nette augmentation du nombre de stratégies socio-affectives par rapport aux résultats antérieurs pendant la phase de présentation à l'oral. Ceci est dû au fait que les conseils donnés à autrui sont souvent de ce type. De plus, l'évolution des stratégies pour cette phase diffère, et pour l'approche globale des deux autres phases, et pour les résultats globaux. 
Figure 5. Approche globale des tâches. Utilisation de techniques d'aide et de conseil

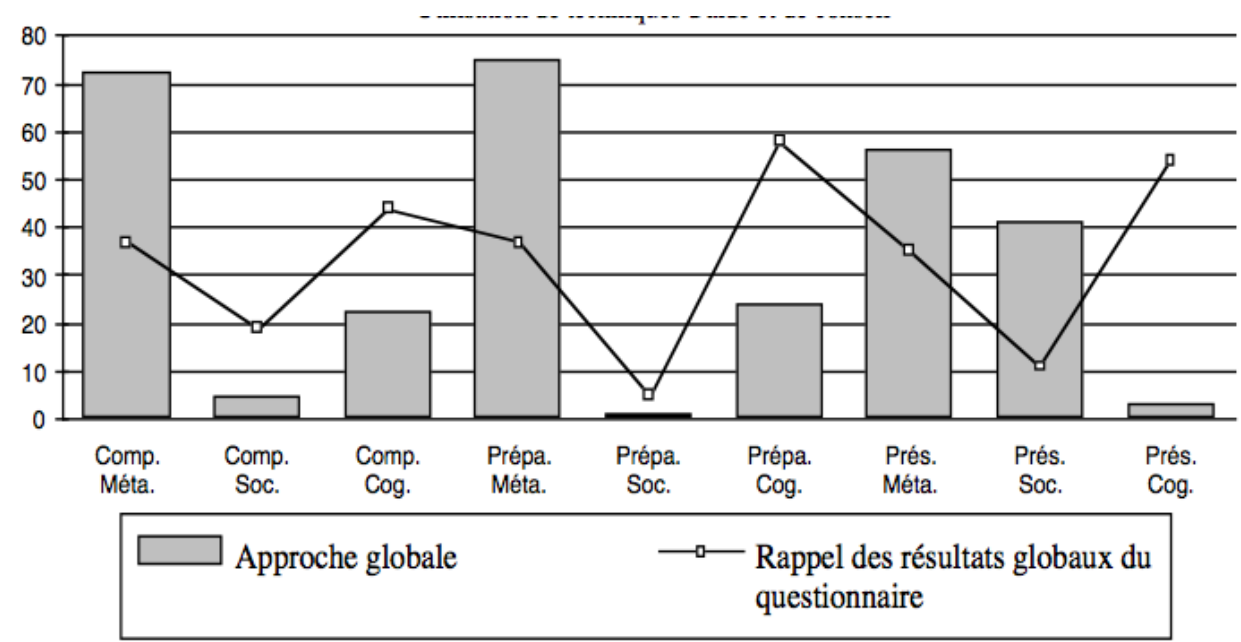

Une dernière analyse nous semblait intéressante. Nous sommes revenus sur l'utilisation des stratégies cognitives complexes (association, élaboration, contextualisation, image mentale, etc..) lors des phases de préparation et de présentation à l'oral de l'exercice. Si on examine en détail l'utilisation de ces stratégies pour l'approche spécifique du texte (acronymes, unités de mesure, noms propres, etc..) pendant ces phases, quelques pourcentages frappants apparaissent.

En effet, les réponses aux questions sur l'approche spécifique comportaient beaucoup de «appris par cœur» sans plus de précisions. Cette réponse n'indique pas de démarche particulière et nous les avons classés dans une stratégie cognitive appelée "mémorisation" qui est aussi vague que la réponse. Ensuite nous avons calculé les pourcentages qui se trouvent dans la figure 6 en faisant abstraction des «appris par cœur » afin de nous concentrer sur les réponses faisant état de démarches particulières.

On peut y noter une proportion élevée des stratégies complexes telles l'élaboration, l'association ou l'image mentale pour l'approche spécifique par rapport aux stratégies plus simples au plan cognitif comme la répétition, la traduction ou le transfert, surtout pendant la phase de présentation à l'oral pour laquelle les chiffres atteignent parfois $100 \%$.

Figure 6. Utilisation de stratégies cognitives complexes pour l'approche spécifique

\begin{tabular}{|l|l|l|}
\hline & Préparation de l'oral & Présentation à l'oral \\
\hline Mots & $33 \%$ & $82 \%$ \\
\hline Acronymes & $50 \%$ & $100 \%$ \\
\hline Unités de mesure & $41 \%$ & $100 \%$ \\
\hline Nombres et dimensions & question non posée & $83 \%$ \\
\hline Noms propres & question non posée & $67 \%$ \\
\hline
\end{tabular}




\section{Conclusion} ce type de stratégie soit largement majoritaire pendant la phase de présentation du moment où les apprenants ont pu expliciter leur démarche nous fait penser qu'elles ont une place importante dans l'exploitation de ce type de texte quand il s'agit de reproduire des données nouvellement acquises.

\section{BIBLIOGRAPHIE}

Chamot, Anna Uhl et Lisa Kupper. 1989. « Learning strategies in foreign language instruction ». Foreign Language Annals 22/1, 13-24.

Gardner, R.C. et P.D. MacIntyre. 1992. « A student's contributions to second language learning. Part 1: Cognitive variables ». Language Teaching 25/3, 139-150.

Narcy, Jean-Paul. 1990. Apprendre une langue étrangère. Paris : Les Éditions d'Organisation. 
O'Malley, J. Michael et Anna Uhl Chamot. 1990. Learning Strategies in Second Language Acquisition. Cambridge : Cambridge University Press.

Oxford, R.L. et D. Crookall. 1989. « Research on language learning strategies: methods, findings and instructional issues ». Modern Language Journal 75, 404-19.

\section{ANNEXES}

\section{Annexe 0 : Compte rendu des discussions}

La discussion qui a suivi cette communication a porté sur deux points : l'aspect pédagogique du travail en classe et l'analyse de l'utilisation des stratégies d'apprentissage.

Nous avons tenu compte des questions posées sur le déroulement de l'exercice dans la version écrite de la communication. Pour ce qui est de l'utilité de l'exercice dans l'apprentissage, à notre avis, la préparation faite en classe avec l'enseignant a aidé les étudiants à être plus efficaces et leur performance lors de la présentation orale était améliorée du fait qu'ils n'étaient pas embarrassés par des notes écrites. Par ailleurs, les étudiants pouvaient prendre connaissance de leurs lacunes grâce à la grille de correction communiquée après l'exercice.

Pour ce qui est des suites pédagogiques, nous n'avons pas encore eu le temps de refaire l'exercice en tenant compte des résultats trouvés. Par ailleurs, le but du travail n'était pas d'engendrer d'autres exercices sur la présentation de textes à l'oral, mais d'observer et analyser les stratégies d'apprentissage pour une tâche donnée.

Nous envisageons ultérieurement de collecter les données utiles en questionnant les apprenants individuellement. On nous a aussi demandé si les recherches citées tenaient compte du niveau socio-culturel des apprenants - ce qui aurait pu expliquer l'utilisation de stratégies cognitives complexes par des étudiants. Nous avons répondu que les travaux faisaient surtout état de différences dans l'utilisation des stratégies à différents niveaux d'apprentissage. Enfin, nous n'avons pas examiné la liaison entre le niveau des textes étudiés et les stratégies cognitives choisies. Il serait effectivement intéressant de rapprocher les notes obtenues par les étudiants et les stratégies utilisées.

Annexe 1 : Exemple de texte utilisé pour l'exercice 


\section{VIRTUAL REALITY PACKAGE} $P(\mathrm{~S} / S \mathrm{~S} / \mathrm{N}$ morkstations/silicon graphics

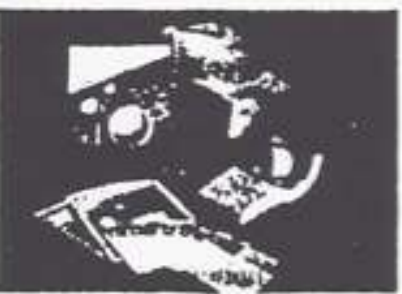

WorfdToulkit is a virual reality developes's package for l'e's, Silicon (iraphies and SIIN Work. slations. A wide range of npplication arras are covored, including onginnoring. archifecture. re. search and development. $\mathrm{C} A \mathrm{D} / \mathrm{C} \wedge \mathrm{M}$. chemical enginerring and design. Worlditoolkit is a library of functions used to buildi virtual reality applicatiens WTK provides a hardwaro independen! system for prototyping. building and delivery realtime

Virtual Presence I.td. 25 Corsham St. Iondon NI (6) R. UK.

Trl $+44-71-2539699$

liax $+44-7 \mid .49(1) 8968$

\section{FAST INFO: ENTER IEN 2300 ON READER CARD}

I. Find the following information about your product:

Name of the product

Manufacturer

Generic name (what it is)

Function (what it does)

Characteristics

Physical description

Capabilities

Compatibility with other products

Accessories available, etc.

II. <\#GRAS\#></\#GRAS\#>Make a list of the following things for your text:

Active verbs

Passive verbs

Verbs ending in -ing

Descriptive adjectives

Noun groups of more than two words

Acronyms

\section{Annexes 2 à 4 (voir fichier)}

\section{RÉSUMÉS}

Après avoir choisi un court texte en anglais présentant un produit informatique, nos étudiants en IUT doivent le lire et en faire une présentation orale de deux à trois minutes sans notes. Ces textes comportent une haute concentration d'acronymes et de termes techniques qui doivent être compris, retenus et reproduits. Nous avons demandé aux étudiants (sous forme de questionnaire) d'expliquer les stratégies qu'ils utilisent lors de ce travail. Cette communication a 
pour but la présentation et l'analyse de ces stratégies afin de chercher une spécificité dans leur utilisation pour ce type de texte.

After choosing a short text in English presenting a computer product, our students must read it and prepare a short oral presentation of the product to be done without using notes. These texts contain many acronyms and other technical terms which must be understood, retained and reproduced. We asked the students to respond to a questionnaire on the strategies they used during this exercise. This paper presents and analyses these strategies in order to ascertain if specific strategies are used for this type of text.

INDEX

Mots-clés : anglais de spécialité, cognition, stratégie d'apprentissage

Keywords : cognition, ESP, learning strategy

\section{AUTEUR}

JANET ATLAN

IUT de Nancy 2, équipe COSTECH-UTC. janetatlan@gmail.com 